Article

\title{
Effects of Dietary Zeolite Supplementation as an Antibiotic Alternative on Growth Performance, Intestinal Integrity, and Cecal Antibiotic Resistance Genes Abundances of Broilers
}

\author{
Hengman Qu, Yefei Cheng, Yueping Chen, Jun Li, Yurui Zhao and Yanmin Zhou * \\ College of Animal Science and Technology, Nanjing Agricultural University, No. 6, Tongwei Road, Xuanwu \\ District, Nanjing 210095, China; qhm13457@163.com (H.Q.); 2017205020@njau.edu.cn (Y.C.); \\ chenyp0321@163.com (Y.C.); 2017105070@njau.edu.cn (J.L.); 2017105068@njau.edu.cn (Y.Z.) \\ * Correspondence: zhouym6308@163.com; Tel.: +86-25-8439-6067
}

Received: 1 October 2019; Accepted: 28 October 2019; Published: 1 November 2019

Simple Summary: The widespread use of antibiotics in livestock production has attracted public attention due to antibiotic resistance. It is necessary to search for antibiotic alternatives in feeds. Zeolite, a kind of feed additive or feed raw material, can enhance growth performance, balance intestinal bacteria, and improve antioxidant capacity in animals. In vitro studies showed zeolite could reduce antibiotic resistance genes (ARGs) abundances. This research compared the effects of antibiotics and zeolite on growth performance, intestinal morphology and barrier function, and cecal ARGs abundances of broilers. Our results indicated that dietary zeolite supplementation exerted similar or better effects than antibiotic inclusion.

\begin{abstract}
The study investigated the effects of dietary zeolite supplementation as an antibiotic alternative on growth performance, intestinal integrity, and cecal antibiotic resistance genes abundances of broilers. One-day-old chicks were assigned into three groups and fed a basal diet or a basal diet supplemented with antibiotics $(50 \mathrm{mg} / \mathrm{kg})$ or zeolite $(10 \mathrm{~g} / \mathrm{kg})$. Antibiotic or zeolite increased $(p<0.05)$ average daily gain (ADG) from 1 to 42 days and duodenal villus height to crypt depth ratio (VH:CD) at 21 days. Zeolite increased $(p<0.05)$ ADG and average daily feed intake from 1 to 21 days, jejunal VH:CD at 21 and 42 days, ileal VH and VH:CD at 42 days, zonula occludens- 1 mRNA abundance at 21 days, and duodenal occludin mRNA abundance at 42 days, whereas reduced $(p<0.05)$ jejunal CD and malondialdehyde levels in ileum at 21 days and duodenum at 42 days, serum D-lactic acid and diamine oxidase levels at 42 days, and plasma lipopolysaccharide content at 21 and 42 days. Antibiotics reduced $(p<0.05)$ duodenal claudin- 2 mRNA abundance at 21 days, whereas increased $(p<0.05)$ cecal tet $B$ abundance at 42 days. These findings suggested that the beneficial effects of zeolite in broilers were more pronounced than that of antibiotics.
\end{abstract}

Keywords: zeolite; antibiotic; growth performance; intestinal barrier; antibiotic resistance genes; broilers

\section{Introduction}

The widespread use of in-feed antibiotics has arisen into a controversial issue worldwide and is facing reduced social acceptance due to the public health problem, such as antibiotic residue and antibiotic resistant bacteria [1-4]. The production of antibiotic resistance genes (ARGs) is an important reason for bacterial resistance and has become an emerging environmental contaminant [5]. It was investigated that more than $95 \%$ Escherichia coli isolates from sick chickens were resistant to at least 
one antibiotic between 1993 and 2013 in China [6]. Thus, using antibiotic growth promoters has been eliminated or limited. On 1 January 2006, the European Union withdrew the approval for antibiotics as growth promoters [7]. Recently, the Ministry of Agriculture of China will prohibit the use of all kinds of antibiotics as growth promoters from 1 January 2020 [8]. As a result, it is necessary to find potential alternatives to in-feed antibiotics in animals' production.

Zeolite is crystalline, hydrated aluminosilicates of alkali and alkaline earth cations, which has a large number of regularly arranged, uniform size, interlinked pores and channels, large specific surface area, strong adsorption, ion exchangeability, and good heat and acid resistance [9-11]. These characteristics of zeolite endow its extensive applications in various industries, including metallurgy [12], pesticide [13], environmental protection [14,15], and livestock production [16,17]. As a kind of feed additive or feed raw material, several researches have shown that zeolite promoted growth performance [10,18-20], improved digestive function [20,21], absorbed harmful substances in digestive tract [20,22], benefited intestinal microecology and villus development [23,24], and enhanced antioxidant capacity [16,17] in broilers and/or pigs. Interestingly, it is reported that zeolite exerted a similar effect of antibiotics, efficiently working against the gram-negative bacteria [25-28]. Moreover, in vitro studies have also shown that zeolite can absorb antibiotics strongly $[29,30]$ and even reduce the abundances of some ARGs in sludge compost [31]. Poultry production is currently perceived as one of the major sources of pathogens and antimicrobial resistance [32]. However, whether zeolite can reduce in vivo ARGs produced during growth and development in broilers is still unknown. Based on the aforementioned, we speculated that dietary zeolite supplementation might be an additive comparable to antibiotics in feed. The current study was, therefore, designed to evaluate the effects of zeolite and antibiotics on growth performance, intestinal barrier function and integrity, and ARGs abundance in the cecal contents of broilers, and hence to investigate whether dietary zeolite supplementation can be an additive that could produce more pronounced beneficial consequences than antibiotics used in broilers diets.

\section{Materials and Methods}

\subsection{Animals, Diets, and Treatments}

All experimental conditions and animal procedures were approved by the Nanjing Agricultural University Institutional Animal Care and Use Committee (Certification No. SYXK (Su) 2017-0007).

A total of 144,1-day-old male Arbor Acres broilers with similar hatching weight were obtained from a commercial hatchery and raised from 1 to 42 days. The chicks were randomly distributed into 3 dietary treatments consisting of 6 replicates. A replicate included a cage with 8 chicks. The chicks in 3 treatments were received a corn-soybean meal basal diet (CON), and the basal diet supplemented with either $50 \mathrm{mg} / \mathrm{kg}$ chlortetracycline (ANT, Jinhe Biotechnology Co. Ltd. Hohhot, China) or 10 $\mathrm{g} / \mathrm{kg}$ zeolite (ZEO), which was kindly gifted by the Beipiao Xinmingshuang Zeolite Agency (Beipiao, China). Using a dosage at $10 \mathrm{~g} / \mathrm{kg}$ was according to these studies that showed that $10 \mathrm{~g} / \mathrm{kg}$ zeolite supplementation exhibited beneficial consequences in improving the growth performance and intestinal flora composition of broilers [33-35]. Ingredient compositions and nutrient contents of the basal diet are the same as the study of Cheng et al. [36]. Birds were allowed free access to water and mash feed in 3-level cages $(120 \mathrm{~cm} \times 60 \mathrm{~cm} \times 50 \mathrm{~cm})$ in a room with controlled environmental conditions. Continuous light in the room was provided during the whole experimental period. The temperature of the room was maintained at 32 to $34{ }^{\circ} \mathrm{C}$ for the first 3 days and then reduced by 2 to $3{ }^{\circ} \mathrm{C}$ per week to a final temperature of $20^{\circ} \mathrm{C}$. At 21 and 42 days of age, birds were weighed after a 12-h feed withdrawal, and feed intake was recorded by replicate (cage) to calculate average daily gain (ADG), average daily feed intake (ADFI), and the feed/gain ratio (F/G). Birds that died during the experimental period were weighed, and the data were included in the calculation of $\mathrm{F} / \mathrm{G}$. 


\subsection{Sample Collection}

At days 21 and 42 of the experiment, 18 male birds (one bird close the average body weight of each replicate) were selected. Whole blood samples were collected in both glass anti-coagulant tubes coated with heparin sodium (Xiamen Bioendo Technology Co., Ltd. Xiamen, China) and tubes without anti-coagulant by wing vein puncture. Plasma separated from blood samples collected in anti-coagulant tubes after centrifugation at $3000 \times \mathrm{g}$ for $3 \mathrm{~min}$ at $4{ }^{\circ} \mathrm{C}$. Serum was separated from blood samples collected in tubes without anti-coagulant after centrifugation at $4450 \times \mathrm{g}$ for $15 \mathrm{~min}$ at $4{ }^{\circ} \mathrm{C}$. The plasma and serum were immediately frozen at $-20^{\circ} \mathrm{C}$ until subsequent analysis. Then, birds were euthanatized by cervical dislocation and necropsied immediately. The whole gastrointestinal tract was rapidly removed and separated. About $2 \mathrm{~cm}$ segments of mid-duodenum, mid-jejunum, and mid-ileum were then harvested, flushed repeatedly with ice-cold PBS (pH 7.4), and immediately immersed in $10 \%$ fresh, chilled paraformaldehyde solution for subsequent histological measurement. Then, the mucosa of duodenum, jejunum, and ileum was collected and rapidly frozen in liquid nitrogen and stored at $-80^{\circ} \mathrm{C}$ for further analysis. The cecum samples (left side) were excised aseptically, and the contents were removed to a sterile cryogenic vial rapidly and frozen in liquid nitrogen and stored at $-80^{\circ} \mathrm{C}$ for further analysis.

\subsection{Morphological Examination}

Intestinal segments were dehydrated, cleared, and embedded in paraffin. Serial sections were then cut at $5 \mu \mathrm{m}$, deparaffinized in xylene, rehydrated, and stained with hematoxylin and eosin. Histological slides were prepared from 3 cross-sections of each intestinal sample. A total of 10 intact, well-oriented crypt-villus and associated crypts were measured from each segment. Villus height and crypt depth were measured using a Nikon ECLIPSE 80i light microscope equipped with a computer-assisted morphometric system (Nikon Corporation, Tokyo, Japan). All staining chemicals were sourced from Sigma-Aldrich Chemical (St Louis, MO, USA).

\subsection{Analysis of Mucosal Antioxidant Parameters}

Approximately $0.3 \mathrm{~g}$ of duodenal, jejunal, and ileal mucosa samples were homogenized (1:9, $\mathrm{wt} / \mathrm{vol}$ ) with ice-cold $154 \mathrm{mmol} / \mathrm{L}$ sodium chloride solution using an Ultra-Turrax homogenizer (Tekmar $\mathrm{Co}$, Cincinnati, OH, USA) and then centrifuged at $4450 \times \mathrm{g}$ for $15 \mathrm{~min}$ at $4{ }^{\circ} \mathrm{C}$. The supernatant was then collected and stored at $-20^{\circ} \mathrm{C}$ for assaying mucosal antioxidant and immune parameters. Total protein concentration, malondialdehyde (MDA) content, total antioxidant capacity (T-AOC), and total superoxide dismutase (T-SOD) activity were measured using diagnostic kits (Nanjing Jiancheng Bioengineering Institute, Nanjing, China) according to the manufacturer's instructions. All results were normalized against total protein concentration in each sample for inter-sample comparison.

\subsection{Measurement of Bacterial Lipopolysaccharide (LPS) Content in Plasma and D-Lactic Acid Level and Diamine Oxidase Activity (DAO) in Serum}

The plasma LPS content was determined by a chromogenic matrix Limulus kit (Xiamen Bioendo Technology Co., Ltd. Xiamen, China) according to the manufacture's instruction. The serum D-lactic acid concentration was quantified using a D-lactic acid colorimetric assay kit (BioVision Inc. Shanghai, China) on the basis of the manufacturer's protocols. The activity of DAO in the serum was determined by a corresponding reagent kit (Nanjing Jiancheng Bioengineering Institute, Nanjing, China).

\subsection{Messenger RNA Quantitative PCR}

Total RNA from intestinal mucosa was isolated using Trizol reagent according to the instructions of the manufacturer (TaKaRa Biotechnology Co. Ltd., Dalian, China). The final concentration and purity of RNA was quantified using a NanoDrop ND-1000 UV spectrophotometer (Nano Drop Technologies, Wilmington, DE, USA) from OD260/280 readings obtaining ratios between 1.8 and 2.1. RNA samples 
were then diluted with diethyl pyrocarbonate-treated water (Biosharp) to a final concentration of $0.5 \mu \mathrm{g} / \mu \mathrm{L}$. After that, $1 \mu \mathrm{g}$ of total RNA was reversed immediately following the RNA isolation using the Prime Script TM RT reagent kit (TaKaRa Biotechnology Co., Ltd., Dalian, China). The primer sequences were the same with our present study [37] and synthesized by Invitrogen Biotechnology Co., Ltd. (Shanghai, China). The cDNA samples were amplified with the TB Green ${ }^{\mathrm{TM}}$ Premix Ex

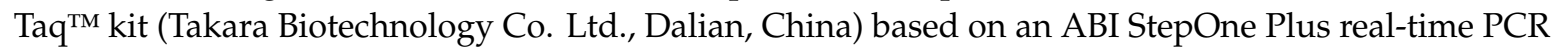
system (Applied Biosystems, Foster City, CA, USA). Detailed procedures of real-time quantitative PCR were performed following the descriptions by our present study [37]. The gene expression level was calculated relative to $\beta$-actin using the $2^{-\Delta \Delta C T}$ method [38].

\subsection{DNA Extraction and Quantitative PCR ( $q P C R$ )}

DNA was extracted from $0.2 \mathrm{~g}$ of each cecal contents sample using the QIAamp Fast DNA Stool Mini Kit for feces (QIAGEN GmbH, Hilden, Germany) according to the manufacturer's instructions. The $16 \mathrm{~S}$ rRNA gene and 11 tetracycline resistance genes $(\operatorname{tet} A$, tet $B$, tet $C$, tet $E$, tet $G$, tet $M$, tet $O$, tet $Q$, tet $T$, tet $W$, and tet $X$ ) were detected and analyzed in this study. All qPCR assays were performed using the TB Green ${ }^{\mathrm{TM}}$ Premix Ex Taq ${ }^{\mathrm{TM}}$ kit (Takara Biotechnology Co. Ltd., Dalian, China) on the ABI StepOne Plus real-time PCR system (Applied Biosystems, Foster City, CA, USA). The qPCR mixture of the 11 genes were in a total volume of $10 \mu \mathrm{L}$, containing $5 \mu \mathrm{L}$ TB Green Premix Ex Taq II (TliRNaseH Plus) $(2 \times)$ (Takara Biotech, Co., Ltd., Dalian, China), $0.4 \mu \mathrm{L}$ of each primer (primer sequences are referenced the study of Zhu et al. [39]), $0.2 \mu \mathrm{L}$ ROX Reference Dye (50×), and $1 \mu \mathrm{L}$ of DNA template, and the rest of the volume was made up of distillation-distillation $\mathrm{H}_{2} \mathrm{O}$. The thermal cycling steps for quantitative PCR amplification were as follows: (1) $95^{\circ} \mathrm{C}$ for $30 \mathrm{~s}$; (2) $95{ }^{\circ} \mathrm{C}$ for $5 \mathrm{~s}$; (3) annealing temperature at $60{ }^{\circ} \mathrm{C}$ for $30 \mathrm{~s}$, where steps (2) to (3) were repeated 40 times. The relative abundances of ARGs were calculated relative to $16 \mathrm{~S}$ rRNA using the $2^{-\Delta \Delta C T}$ method [38].

\subsection{Statistical Analysis}

Data were analyzed by one-way analysis of variance (ANOVA) using the SPSS statistical software (Ver.16.0 for windows, SPSS Inc., Chicago, IL, USA) with a pen (cage) as the experimental unit. Differences among treatments were examined using the Tukey-Kramer's multiple range tests, which were considered significant when the $p$-value was less than 0.05 . The means and standard errors of means (SEM) were presented.

\section{Results}

\subsection{Growth Performance}

Compared with the CON group (Table 1), dietary zeolite supplementation increased the ADG and ADFI of broilers from 1 to 21 days $(p<0.05)$. Moreover, dietary either zeolite or antibiotic supplementation improved ADG of broilers from 1 to 42 days $(p<0.05)$, with the values of ADG being similar between the two treatments $(p>0.05)$. However, treatments did not affect growth performance from 22 to 42 days $(p>0.05)$.

\subsection{Intestinal Morphology}

Compared with the CON group (Table 2), birds fed either zeolite or antibiotic had a higher duodenal VH:CD at 21 days $(p<0.05)$. Dietary zeolite supplementation increased $(p<0.05)$ ileal VH at 42 days, VH:CD in the jejunum at both 21 and 42 days, and ileum at 42 days, whereas decreased $(p<$ 0.05 ) jejunal $C D$ at 42 days. Additionally, dietary antibiotic supplementation reduced jejunal CD at 21 days when in comparison with the CON group $(p<0.05)$; however, zeolite inclusion did not exert such effect $(p>0.05)$. 
Table 1. Effects of zeolite and antibiotic supplementation on growth performance in broilers.

\begin{tabular}{cccccc}
\hline Items $^{\mathbf{1 , 2}}$ & CON & ANT & ZEO & SEM & $p$-Value \\
\hline 1-21 days & & & & & \\
ADG (g/d) & $26.04^{\mathrm{b}}$ & $28.01^{\mathrm{ab}}$ & $30.26^{\mathrm{a}}$ & 0.716 & 0.009 \\
ADFI (g/d) & $39.98^{\mathrm{b}}$ & $41.84^{\mathrm{ab}}$ & $45.07^{\mathrm{a}}$ & 0.743 & 0.008 \\
F/G (g/g) & 1.54 & 1.50 & 1.49 & 0.016 & 0.495 \\
22-42 days & & & & & \\
ADG (g/d) & 73.43 & 77.17 & 75.64 & 1.131 & 0.423 \\
ADFI (g/d) & 155.13 & 159.09 & 151.54 & 2.348 & 0.428 \\
F/G (g/g) & 2.05 & 2.07 & 2.01 & 0.028 & 0.657 \\
1-42 days & & & & & \\
ADG (g/d) & $49.73^{\mathrm{b}}$ & $52.58^{\mathrm{a}}$ & $52.80^{\mathrm{a}}$ & 0.529 & 0.019 \\
ADFI (g/d) & 93.68 & 97.91 & 96.95 & 1.153 & 0.308 \\
F/G (g/g) & 1.88 & 1.86 & 1.84 & 0.017 & 0.591
\end{tabular}

a, b Means within a row with different superscripts differ significantly at $p<0.05 .{ }^{1}$ ADG, average daily gain; ADFI, average daily feed intake; $\mathrm{F} / \mathrm{G}$, feed to gain ratio. ${ }^{2} \mathrm{CON}$ : broilers receiving a corn-soybean meal basal diet without antibiotic and zeolite; ANT: broilers receiving the basal diet supplemented with $50 \mathrm{mg} / \mathrm{kg}$ chlortetracycline; ZEO: broilers were given the basal diet supplemented with $10 \mathrm{~g} / \mathrm{kg}$ zeolite. SEM, standard error of means $(n=6)$.

Table 2. Effects of zeolite and antibiotic supplementation on intestinal integrity in broilers.

\begin{tabular}{cccccc}
\hline Items ${ }^{1,2}$ & CON & ANT & ZEO & SEM & $p$-Value \\
\hline 21 days & & & & \\
Duodenum & & & & \\
VH $(\mu \mathrm{m})$ & 1661.18 & 1728.58 & 1663.85 & 42.077 & 0.782 \\
CD $(\mu \mathrm{m})$ & $232.44^{2}$ & 190.98 & 184.59 & 9.255 & 0.063 \\
VH:CD & $7.26^{\mathrm{b}}$ & $9.11^{\mathrm{a}}$ & $9.25^{\mathrm{a}}$ & 0.347 & 0.021 \\
Jejunum & & & & \\
VH $(\mu \mathrm{m})$ & 1282.88 & 1182.81 & $1441.12^{\mathrm{ab}}$ & 52.896 & 0.131 \\
CD $(\mu \mathrm{m})$ & $274.70^{\mathrm{a}}$ & $212.79^{\mathrm{b}}$ & $231.84^{\mathrm{ab}}$ & 0.820 & 0.046 \\
VH:CD & $4.73^{\mathrm{b}}$ & $5.55^{\mathrm{ab}}$ & $6.38^{\mathrm{a}}$ & 0.262 & 0.026 \\
Ileum & & & & \\
VH $(\mu \mathrm{m})$ & 825.50 & 789.28 & 748.92 & 40.177 & 0.762 \\
CD $(\mu \mathrm{m})$ & 159.77 & 147.93 & 152.37 & 8.823 & 0.872 \\
VH:CD & 5.32 & 5.82 & 4.91 & 0.301 & 0.491 \\
42 days & & & & \\
Duodenum & & & & \\
VH $(\mu \mathrm{m})$ & 1922.33 & 1752.94 & 1841.45 & 33.717 & 0.118 \\
CD $(\mu \mathrm{m})$ & 239.03 & 207.98 & 207.25 & 7.190 & 0.116 \\
VH:CD & 8.16 & 8.54 & 8.89 & 0.215 & 0.406 \\
Jejunum & & & & \\
VH $(\mu \mathrm{m})$ & 1353.73 & 1464.09 & 1410.14 & 48.407 & 0.675 \\
CD $(\mu \mathrm{m})$ & $194.61^{\mathrm{a}}$ & $173.01^{\mathrm{ab}}$ & $131.97^{\mathrm{b}}$ & 10.082 & 0.024 \\
VH:CD & $7.18^{\mathrm{b}}$ & $8.51^{\mathrm{b}}$ & $10.69^{\mathrm{a}}$ & 0.410 & $<0.001$ \\
Ileum & & & & \\
VH $(\mu \mathrm{m})$ & $824.86^{\mathrm{b}}$ & $863.51^{\mathrm{ab}}$ & $1057.39^{\mathrm{a}}$ & 38.246 & 0.018 \\
CD $(\mu \mathrm{m})$ & 131.93 & 117.62 & 115.79 & 3.852 & 0.177 \\
VH:CD & $6.25^{\mathrm{b}}$ & $7.52^{\mathrm{ab}}$ & $9.19^{\mathrm{a}}$ & 0.405 & 0.004 \\
\hline
\end{tabular}

a, b Means within a row with different superscripts differ significantly at $p<0.05 .{ }^{1} \mathrm{VH}$, villus height; CD, crypt depth; VH:CD, the ratio of villus height to crypt depth. ${ }^{2} \mathrm{CON}$ : broilers receiving a corn-soybean meal basal diet without antibiotic and zeolite; ANT: broilers receiving the basal diet supplemented with $50 \mathrm{mg} / \mathrm{kg}$ chlortetracycline; ZEO: broilers were given the basal diet supplemented with $10 \mathrm{~g} / \mathrm{kg}$ zeolite. SEM, standard error of means $(n=6)$.

\subsection{Antioxidant Function of Intestinal Mucosa}

As shown in Table 3, compared with the CON group, dietary zeolite supplementation reduced $(p<0.05)$ MDA content in the ileum at 21 days and duodenum at 42 days. However, this effect was not 
observed by antibiotic inclusion $(p>0.05)$. Antioxidant enzyme activities were similar among groups $(p>0.05)$.

Table 3. Effects of zeolite and antibiotic supplementation on antioxidant function of intestinal mucosa in broilers.

\begin{tabular}{|c|c|c|c|c|c|}
\hline Items 1,2 & CON & ANT & ZEO & SEM & $p$-Value \\
\hline \multicolumn{6}{|l|}{21 days } \\
\hline \multicolumn{6}{|l|}{ Duodenum } \\
\hline MDA (nmol/mg protein) & 0.73 & 0.62 & 0.64 & 0.059 & 0.740 \\
\hline T-AOC (U/ mg protein) & 0.55 & 0.46 & 0.63 & 0.032 & 0.103 \\
\hline $\begin{array}{l}\text { T-SOD (U/mg protein) } \\
\text { Jejunum }\end{array}$ & 190.17 & 190.28 & 224.43 & 7.684 & 0.125 \\
\hline MDA (nmol/mg protein) & 1.03 & 0.77 & 0.71 & 0.059 & 0.054 \\
\hline T-AOC (U/mg protein) & 0.56 & 0.61 & 0.65 & 0.020 & 0.212 \\
\hline $\begin{array}{l}\text { T-SOD (U/mg protein) } \\
\text { Ileum }\end{array}$ & 190.61 & 190.08 & 199.62 & 2.717 & 0.389 \\
\hline MDA (nmol/mg protein) & $1.30^{\mathrm{a}}$ & $1.34^{\mathrm{a}}$ & $0.73^{\mathrm{b}}$ & 0.093 & 0.006 \\
\hline T-AOC (U/mg protein) & 1.01 & 1.03 & 1.16 & 0.058 & 0.541 \\
\hline \multicolumn{6}{|l|}{42 days } \\
\hline \multicolumn{6}{|l|}{ Duodenum } \\
\hline MDA (nmol/mg protein) & $1.32^{\mathrm{a}}$ & $1.08^{\mathrm{a}}$ & $0.76^{\mathrm{b}}$ & 0.071 & 0.001 \\
\hline T-AOC (U/ mg protein) & 0.87 & 0.89 & 0.90 & 0.035 & 0.944 \\
\hline $\begin{array}{l}\text { T-SOD (U/mg protein) } \\
\text { Jejunum }\end{array}$ & 211.36 & 215.05 & 213.97 & 6.410 & 0.974 \\
\hline MDA (nmol/mg protein) & 0.71 & 0.64 & 0.62 & 0.044 & 0.733 \\
\hline T-AOC (U/ mg protein) & 0.78 & 0.78 & 0.83 & 0.030 & 0.791 \\
\hline T-SOD (U/mg protein) & 225.15 & 238.01 & 229.00 & 7.015 & 0.758 \\
\hline \multicolumn{6}{|l|}{ Ileum } \\
\hline MDA (nmol/mg protein) & 0.95 & 0.72 & 0.80 & 0.049 & 0.240 \\
\hline T-AOC (U/mg protein) & 1.09 & 1.02 & 0.92 & 0.037 & 0.173 \\
\hline SOD (U/mg protein) & 158.02 & 161.10 & 155.40 & 2.486 & 0.696 \\
\hline
\end{tabular}

a, b Means within a row with different superscripts differ significantly at $p<0.05 .{ }^{1}$ MDA, malondialdehyde; T-SOD, total superoxide dismutase; T-AOC, total antioxidant capacity. ${ }^{2}$ CON: broilers receiving a corn-soybean meal basal diet without antibiotic and zeolite; ANT: broilers receiving the basal diet supplemented with $50 \mathrm{mg} / \mathrm{kg}$ chlortetracycline; ZEO: broilers were given the basal diet supplemented with $10 \mathrm{~g} / \mathrm{kg}$ zeolite. SEM, standard error of means $(n=6)$.

\subsection{Plasma LPS Content and Serum DAO and D-Lactic Levels}

Compared with the CON group (Table 4), dietary zeolite supplementation reduced $(p<0.05)$ plasma LPS content at 21 days and serum DAO and D-lactic acid level at 42 days, whereas the values of these indexes were not affected by antibiotic supplementation $(p>0.05)$. Moreover, dietary zeolite supplementation decreased $(p<0.05)$ plasma LPS concentration at 42 days compared with the ANT group.

\subsection{Gene Expression Levels Related to Intestinal Barrier Function}

Broilers receiving zeolite exhibited higher mRNA abundances of zonula occludens-1 (ZO-1) in the jejunum at 21 days and occludin $(O C L N)$ in the duodenum at 42 days compared with those fed the basal diet $(p<0.05)$. In contrast, dietary antibiotic supplementation reduced claudin-2 (CLDN2) gene expression level in the duodenum at 21 days $(p<0.05)$. Treatments did not affect intestinal mucosal claudin-3 (CLDN3) mRNA abundance $(p>0.05$, Table 5$)$. 
Table 4. Effects of zeolite and antibiotic supplementation on intestinal barrier function in broilers.

\begin{tabular}{cccccc}
\hline Items ${ }^{\mathbf{1 , 2}}$ & CON & ANT & ZEO & SEM & $p$-Value \\
\hline 21 days & & & & & \\
DAO (U/mL) & 18.80 & 17.87 & 18.37 & 0.980 & 0.932 \\
LPS (EU/L) & $0.53^{\mathrm{a}}$ & $0.45^{\mathrm{ab}}$ & $0.32^{\mathrm{b}}$ & 0.032 & 0.014 \\
D-lactic acid (mmol/L) & 2.22 & 2.20 & 2.64 & 0.154 & 0.450 \\
42 days & & & & & \\
DAO (U/mL) & $25.38^{\mathrm{a}}$ & $25.03^{\mathrm{a}}$ & $17.11^{\mathrm{b}}$ & 1.150 & $<0.001$ \\
LPS (EU/L) & $0.32^{\mathrm{ab}}$ & $0.38^{\mathrm{a}}$ & $0.26^{\mathrm{b}}$ & 0.019 & 0.017 \\
D-lactic acid (mmol/L) & $3.83^{\mathrm{a}}$ & $3.64^{\mathrm{a}}$ & $2.64^{\mathrm{b}}$ & 0.186 & 0.009 \\
\hline
\end{tabular}

$\mathrm{a}, \mathrm{b}$ Means within a row with different superscripts differ significantly at $p<0.05 .{ }^{1} \mathrm{DAO}$, diamine oxidase; LPS, bacterial lipopolysaccharide. ${ }^{2} \mathrm{CON}$ : broilers receiving a corn-soybean meal basal diet without antibiotic and zeolite; ANT: broilers receiving the basal diet supplemented with $50 \mathrm{mg} / \mathrm{kg}$ chlortetracycline; ZEO: broilers were given the basal diet supplemented with $10 \mathrm{~g} / \mathrm{kg}$ zeolite. SEM, standard error of means $(n=6)$.

Table 5. Effect of zeolite and antibiotic supplementation on intestinal mucosal gene expression levels related to barrier function in broilers.

\begin{tabular}{|c|c|c|c|c|c|}
\hline Items $^{1,2}$ & $\mathrm{CON}$ & ANT & ZEO & SEM & $p$-Value \\
\hline \multicolumn{6}{|l|}{21 days } \\
\hline \multicolumn{6}{|l|}{ Duodenum } \\
\hline OCLN & 1.00 & 0.83 & 0.79 & 0.061 & 0.327 \\
\hline CLDN2 & $1.00^{\mathrm{a}}$ & $0.61^{b}$ & $1.01^{\mathrm{a}}$ & 0.077 & 0.046 \\
\hline CLDN3 & 1.00 & 0.97 & 1.38 & 0.140 & 0.459 \\
\hline $\mathrm{ZO}-1$ & 1.00 & 0.85 & 0.91 & 0.061 & 0.646 \\
\hline \multicolumn{6}{|l|}{ Jejunum } \\
\hline OCLN & 1.00 & 1.02 & 1.02 & 0.056 & 0.985 \\
\hline CLDN2 & 1.00 & 0.91 & 0.94 & 0.090 & 0.932 \\
\hline CLDN3 & 1.00 & 0.94 & 0.99 & 0.073 & 0.937 \\
\hline $\mathrm{ZO}-1$ & $1.00^{b}$ & $0.95^{\mathrm{b}}$ & $1.52^{\mathrm{a}}$ & 0.080 & 0.001 \\
\hline \multicolumn{6}{|l|}{ Ileum } \\
\hline OCLN & 1.00 & $1.07 \mathrm{~A}$ & 1.14 & 0.133 & 0.923 \\
\hline CLDN2 & 1.00 & 0.96 & 0.90 & 0.105 & 0.936 \\
\hline CLDN3 & 1.00 & 0.91 & 1.20 & 0.199 & 0.843 \\
\hline $\mathrm{ZO}-1$ & 1.00 & 0.87 & 0.99 & 0.059 & 0.653 \\
\hline \multicolumn{6}{|l|}{42 days } \\
\hline \multicolumn{6}{|c|}{ Duodenum } \\
\hline OCLN & $1.00^{b}$ & $1.33^{a b}$ & $1.71^{a}$ & 0.113 & 0.030 \\
\hline CLDN2 & 1.00 & 1.02 & 1.09 & 0.071 & 0.864 \\
\hline CLDN3 & 1.00 & 1.05 & 1.38 & 0.189 & 0.703 \\
\hline ZO-1 & 1.00 & 0.97 & 1.04 & 0.064 & 0.931 \\
\hline \multicolumn{6}{|l|}{ Jejunum } \\
\hline OCLN & 1.00 & 0.92 & 1.06 & 0.077 & 0.788 \\
\hline CLDN2 & 1.00 & 1.06 & 1.11 & 0.133 & 0.947 \\
\hline CLDN3 & 1.00 & 1.19 & 1.27 & 0.161 & 0.801 \\
\hline $\mathrm{ZO}-1$ & 1.00 & 1.09 & 1.16 & 0.097 & 0.821 \\
\hline \multicolumn{6}{|l|}{ Ileum } \\
\hline OCLN & 1.00 & 1.04 & 0.81 & 0.046 & 0.090 \\
\hline CLDN2 & 1.00 & 1.05 & 1.29 & 0.228 & 0.875 \\
\hline CLDN3 & 1.00 & 0.69 & 0.74 & 0.059 & 0.057 \\
\hline $\mathrm{ZO}-1$ & 1.00 & 0.96 & 1.06 & 0.065 & 0.861 \\
\hline
\end{tabular}

a, b Means within a row with different superscripts differ significantly at $\mathrm{p}<0.05 .{ }^{1} C L D N 2$, claudin-2; CLDN3, claudin-3; OCLN, occludin; ZO-1, zonula occludens-1. ${ }^{2} \mathrm{CON}$ : broilers receiving a corn-soybean meal basal diet without antibiotic and zeolite; ANT: broilers receiving the basal diet supplemented with $50 \mathrm{mg} / \mathrm{kg}$ chlortetracycline; ZEO: broilers were given the basal diet supplemented with $10 \mathrm{~g} / \mathrm{kg}$ zeolite. SEM, standard error of means $(n=6)$. 


\subsection{ARGs Detection Frequencies (DFs) in the Cecal Content}

Compared with the CON group (Table 6), AGRs detection frequencies of cecal contents in the ANT group were the highest. The tet $G$, tet $M$, tet $O$, tet $Q$, and tet $W$ were detected in all samples at 21 days. Tet $B$, tet $C$, tet $M$, tet $O$, tet $Q$, tet $W$, and tet $X$ were detected in all samples at 42 days. However, detection frequencies of $t e t A$, tet $E$, and tet $T$ were too low; thus, they were not assayed in the further.

Table 6. Effects of zeolite and antibiotic supplementation on detection frequency (DF) of the 11 targeted tet genes in cecum samples of broilers (\%).

\begin{tabular}{ccccccccc}
\hline \multirow{2}{*}{ Items $^{1}$} & \multicolumn{4}{c}{ 21 Days } & \multicolumn{5}{c}{ 42 Days } \\
\cline { 2 - 9 } & CON & ANT & ZEO & DF & CON & ANT & ZEO & DF \\
\hline Tet $A$ & 0 & 0 & 16.67 & 8.33 & 0 & 0 & 16.67 & 4.16 \\
$\operatorname{Tet} B$ & 50.00 & 100.00 & 100.00 & 87.50 & 100.00 & 100.00 & 100.00 & 100.00 \\
$\operatorname{Tet} C$ & 33.33 & 100.00 & 100.00 & 83.33 & 100.00 & 100.00 & 100.00 & 100.00 \\
$\operatorname{Tet} E$ & 16.67 & 83.33 & 50.00 & 50.00 & 33.33 & 50.00 & 16.67 & 37.50 \\
$\operatorname{Tet} G$ & 100.00 & 100.00 & 100.00 & 100.00 & 50.00 & 100.00 & 66.67 & 66.67 \\
$\operatorname{Tet} M$ & 100.00 & 100.00 & 100.00 & 100.00 & 100.00 & 100.00 & 100.00 & 100.00 \\
$\operatorname{Tet} O$ & 100.00 & 100.00 & 100.00 & 100.00 & 100.00 & 100.00 & 100.00 & 100.00 \\
$\operatorname{Tet} Q$ & 100.00 & 100.00 & 100.00 & 100.00 & 100.00 & 100.00 & 100.00 & 100.00 \\
$\operatorname{Tet} T$ & 50.00 & 66.67 & 16.67 & 45.83 & 50.00 & 50.00 & 83.33 & 58.33 \\
$\operatorname{Tet} W$ & 100.00 & 100.00 & 100.00 & 100.00 & 100.00 & 100.00 & 100.00 & 100.00 \\
$\operatorname{Tet} X$ & 83.33 & 100.00 & 100.00 & 95.83 & 100.00 & 100.00 & 100.00 & 100.00 \\
$\operatorname{DF}$ & 66.67 & 86.36 & 80.30 & 79.17 & 75.76 & 81.82 & 80.30 & 78.79 \\
\hline
\end{tabular}

${ }^{1} \mathrm{CON}$ : broilers receiving a corn-soybean meal basal diet without antibiotic and zeolite; ANT: broilers receiving the basal diet supplemented with $50 \mathrm{mg} / \mathrm{kg}$ chlortetracycline; ZEO: broilers were given the basal diet supplemented with $10 \mathrm{~g} / \mathrm{kg}$ zeolite.

\subsection{Relative Abundances of ARGs in the Cecal Content}

Compared with the CON group, dietary antibiotic supplementation observably increased the relative abundance of tet $B$ in cecal contents of broilers at 42 days $(p<0.05$, Table 7$)$. However, dietary zeolite supplementation did not upregulate ARGs relative abundances in the cecal contents $(p>0.05)$. In contrast, broilers fed zeolite exerted lower relative abundances of $\operatorname{tet} B$, tet $C$, and $\operatorname{tet} X$ in the cecal contents at 42 days when compared with the ANT group $(p<0.05)$.

Table 7. Effects of zeolite and antibiotic supplementation on relative abundances of ARGs in cecum samples of broilers.

\begin{tabular}{cccccc}
\hline Items $^{1}$ & CON & ANT & ZEO & SEM & $p$-Value \\
\hline 21 days & & & & & \\
$\operatorname{Tet} B$ & $1.88 \times 10^{-5}$ & $5.09 \times 10^{-5}$ & $7.97 \times 10^{-5}$ & $3.65 \times 10^{-5}$ & 0.321 \\
$\operatorname{Tet} C$ & $1.42 \times 10^{-5}$ & $2.95 \times 10^{-5}$ & $3.20 \times 10^{-5}$ & $5.66 \times 10^{-6}$ & 0.666 \\
$\operatorname{Tet} G$ & $4.34 \times 10^{-5}$ & $6.98 \times 10^{-5}$ & $5.23 \times 10^{-5}$ & $7.23 \times 10^{-6}$ & 0.335 \\
$\operatorname{Tet} M$ & $9.34 \times 10^{-4}$ & $2.01 \times 10^{-3}$ & $6.46 \times 10^{-4}$ & $2.50 \times 10^{-4}$ & 0.055 \\
$\operatorname{Tet} O$ & $1.96 \times 10^{-1}$ & $1.82 \times 10^{-1}$ & $2.02 \times 10^{-1}$ & $2.28 \times 10^{-2}$ & 0.942 \\
$\operatorname{Tet} Q$ & $1.97 \times 10^{-1}$ & $2.18 \times 10^{-1}$ & $1.48 \times 10^{-1}$ & $3.47 \times 10^{-2}$ & 0.722 \\
$\operatorname{Tet} \mathrm{W}$ & $4.86 \times 10^{-1}$ & $6.02 \times 10^{-1}$ & $5.12 \times 10^{-1}$ & $3.12 \times 10^{-2}$ & 0.294 \\
$\operatorname{Tet} X$ & $8.67 \times 10^{-6}$ & $2.41 \times 10^{-5}$ & $3.45 \times 10^{-5}$ & $6.43 \times 10^{-6}$ & 0.296 \\
$42 \mathrm{days}$ & & & & \\
$\operatorname{Tet} B$ & $3.64 \times 10^{-5} \mathrm{~b}$ & $1.95 \times 10^{-4} \mathrm{a}$ & $1.68 \times 10^{-5} \mathrm{~b}$ & $2.41 \times 10^{-5}$ & $<0.001$ \\
$\operatorname{Tet} \mathrm{C}$ & $2.49 \times 10^{-5} \mathrm{ab}$ & $1.20 \times 10^{-4} \mathrm{a}$ & $9.80 \times 10^{-6} \mathrm{~b}$ & $1.99 \times 10^{-5}$ & 0.034 \\
$\operatorname{Tet} G$ & $1.67 \times 10^{-5}$ & $3.40 \times 10^{-5}$ & $1.72 \times 10^{-4}$ & $3.67 \times 10^{-5}$ & 0.202 \\
$\operatorname{Tet} M$ & $1.76 \times 10^{-3}$ & $2.33 \times 10^{-3}$ & $2.59 \times 10^{-3}$ & $3.42 \times 10^{-4}$ & 0.628 \\
$\operatorname{Tet} \mathrm{O}$ & $2.64 \times 10^{-1}$ & $2.99 \times 10^{-1}$ & $2.10 \times 10^{-1}$ & $2.59 \times 10^{-2}$ & 0.393 \\
$\operatorname{Tet} Q$ & $3.54 \times 10^{-1}$ & $8.07 \times 10^{-1}$ & $6.40 \times 10^{-1}$ & $9.04 \times 10^{-2}$ & 0.114 \\
$\operatorname{Tet} \mathrm{W}$ & $6.47 \times 10^{-1}$ & $9.06 \times 10^{-1}$ & $6.43 \times 10^{-1}$ & $5.23 \times 10^{-2}$ & 0.052 \\
$\operatorname{Tet} X$ & $2.90 \times 10^{-1} \mathrm{ab}$ & $5.74 \times 10^{-1} \mathrm{a}$ & $1.57 \times 10^{-1} \mathrm{~b}$ & $7.13 \times 10^{-2}$ & 0.039 \\
\hline
\end{tabular}

${ }^{1}$ CON: broilers receiving a corn-soybean meal basal diet without antibiotic and zeolite; ANT: broilers receiving the basal diet supplemented with $50 \mathrm{mg} / \mathrm{kg}$ chlortetracycline; ZEO: broilers were given the basal diet supplemented with $10 \mathrm{~g} / \mathrm{kg}$ zeolite. SEM, standard error of means $(n=6)$. 


\section{Discussion}

In this study, broilers fed the basal diet supplemented with zeolite significantly exhibited an increase in ADG and ADFI from days 1 to 21 and ADG from 1 to 42 days, which had better effects than antibiotic supplementation. The improved growth performance of broilers was also demonstrated by Wu et al. [16,17]. Additionally, the dietary inclusion of zeolites can improve ADG and/or feed conversion ratio in pigs [10,20], calves [10], and sheep [10]. The beneficial consequences of zeolite on animal growth performance could be attributed to its binding effect of ammonia [40], absorption of toxins produced during intestinal microbial degradation [41], retarding effect on the digesta transit [10], regulation on digestive enzyme activity [42], and adsorption of mycotoxins [43].

The normal microarchitecture of the small intestine is very crucial in maintaining nutrient absorption and resistance to harmful substrates [44-47]. The VH:CD is considered to be an important criterion for estimating the digestive capacity and absorptive function of the small intestine [16,17]. In the current study, zeolite supplementation exerted a positive influence on intestinal morphology, being embodied in increased VH:CD in the duodenum and jejunum at 21 days, in the jejunum and ileum at 42 days, and $\mathrm{VH}$ in the ileum at 42 days, as well as decreased CD in the jejunum at 42 days. Likewise, it has been previously reported that dietary zeolite supplementation could improve intestinal integrity through increasing $\mathrm{VH}$ in the jejunum and ileum of female broilers [45]. In addition, palygorskite, with similar characteristics to zeolite, was demonstrated by our lab team members that it could enhance intestinal morphology in broilers and laying hens [47-49]. This may because zeolite can adsorb intestinal pathogens bacterium, prevent intestinal mucosa from damage, reinforce the intestinal mucosal barrier, and help in the regeneration of the epithelium [26,50]. Furthermore, the ion-exchange properties of minerals can alter the enzymatic activity of intestinal secretions, which could stimulate the villi and microvilli of intestinal mucosa [51]. Therefore, dietary zeolite supplementation would be an efficient way to improve intestinal development in broilers.

The reactive oxygen species (ROS) are produced during normal metabolism in cells [52]. However, the overproduction of ROS during cellular metabolism could result in oxidative damage [53]. The MDA is the main end-product of the lipid peroxidation caused by ROS, and the accumulation of MDA is usually considered a marker of lipid peroxidation [54]. It is reported that zeolite can decrease the MDA content in the jejunum and ileum of broilers $[16,17]$. Consistently, in the present work, broilers receiving zeolite exhibited decreased MDA content in the ileum at 21 days and in the duodenum at 42 days. The improvement of zeolite on antioxidant capacity in broilers may be partly attributed to its adsorption effect, resulting in reduced oxidative stress by pathogens and other hazardous substances in the gut. In addition, the enhanced intestinal integrity of broilers given zeolite in this study would contribute to a better intestinal health status, which is also a nonnegligible factor for improved antioxidant capacity in broilers.

The DAO is an intracellular enzyme that exists in intestinal epithelial villus cells [55]. Bacterial lipopolysaccharide (LPS) is the major component of the outer surface membrane in almost all gram-negative bacteria [56]. D-lactic acid is a product of bacterial fermentation of carbohydrates, which can be considered as a sensitive marker for reflecting intestinal injury and monitoring intestinal permeability [57]. Decreased DAO activity and contents of D-lactic acid and LPS are usually associated with improved intestinal barrier function of animals [58,59]. In the present study, zeolite supplementation significantly decreased LPS concentrations in plasma at 21 days and DAO activity and D-lactic acid level in serum at 42 days. Tight junctions are the crucial components of the intestinal mucosal barrier. They mainly consist of peripheral membrane protein ZO- 1 and the transmembrane protein OCLN and claudins [60]. Herein, the upregulation of ZO-1, OCLN, and claudins mRNA expression levels would be beneficial to intestinal structure and barrier function. In this work, increased ZO-1 gene expression level in jejunal mucosa at 21 days and OCLN mRNA abundance in duodenal mucosa at 42 days were observed by zeolite supplementation. Those coupled with the simultaneously decreased circulating DAO activity, D-lactic acid, and LPS level, further indicated that zeolite could improve the intestinal barrier function in broilers. Moreover, palygorskite clay with 
similar characteristics was illustrated to enhance intestinal barrier function in broilers and laying hens, as evidenced by decreased serum DAO activity and gene expression levels related to intestinal barrier function [43-45,61]. The improved intestinal morphology and barrier function resulting from zeolite supplementation in the present study indicated that dietary zeolite supplementation could improve intestinal integrity in broilers. On the one hand, zeolite has a strong adsorptive capacity and can adsorb toxic substances and bacteria in the gut that is harmful to the host, eventually excreting them from animals' bodies $[16,17,62,63]$. On the other hand, zeolite has a big specific surface area and allows it to be distributed evenly on the surface of the intestinal mucosa, therefore forming a protective screen to attenuate the gut damage by harmful substances and bacteria [62,64].

The presence of ARGs is the primary reason for bacteria becoming resistant to antibiotics and has become one of the most important public health concerns around the world [5]. Animal intestinal microorganisms live under the pressure of antibiotics when fed a diet supplemented with antibiotics for a long time, and they are prone to develop antibiotic resistance and generate ARGs [1-5]. The results showed that the detection frequencies of tet $A$, tet $E$, and tet $T$ were less than $100 \%$, whereas frequencies of the other eight genes were detected $100 \%$ in the cecal content ( 21 or 42 days), so, we then selected the eight tet genes $(t e t B, t e t C$, tet $G$, tet $M$, tet $O$, tet $Q$, tet $W$, and tet $X)$ for further study. As expected, our experimental results showed that antibiotic inclusion increased the detection rate and relative abundances of ARGs (tet $B$, tet $C$, and tet $X$ ) in the cecal contents of broilers. It is previously demonstrated that zeolite can reduce the abundances of some ARGs in sludge compost in vitro [31]. In this work, dietary supplementation of zeolite significantly decreased cecal tet $B$, tet $C$, and $\operatorname{tet} X$ relative abundances compared with the ANT group, and also numerically reduced their abundances when compared with the CON group. This may be due to its sporous structure and the ability to reduce the selective pressure from heavy metals and the rate of microbe contact, which then causes the horizontal gene transfer through conjugation to reduce [31]. These results indicated that dietary zeolite inclusion could decrease ARGs relative abundances in the cecal content of broilers to some extent, which may be because zeolite can adhere or kill harmful bacteria and herein reducing a possibility of gut bacteria producing ARGs.

\section{Conclusions}

The study suggested that dietary zeolite supplementation had better effects on growth performance, intestinal oxidative status, and integrity in broilers than antibiotic supplementation. Furthermore, zeolite did not increase cecal ARGs abundance. Therefore, the beneficial effects of dietary zeolite supplementation in broilers are more pronounced than the antibiotic used in feed in this study.

Author Contributions: Formal analysis, H.Q., Y.C. (Yefei Cheng), Y.C. (Yueping Chen), J.L., and Y.Z.; writing-original draft preparation, H.Q.; writing-review and editing, Y.C. (Yefei Cheng), Y.C. (Yueping Chen); supervision, Y.Z.

Funding: This research was funded by the National Natural Science Foundation of China, grant number 31872405.

Acknowledgments: The technical assistance of colleagues in our laboratories is gratefully acknowledged. The study was funded by the National Natural Science Foundation of China (Grant No. 31872405).

Conflicts of Interest: The authors declare no conflict of interest in the present work.

\section{References}

1. Singer, R.S.; Finch, R.; Wegener, H.C.; Bywater, R.; Walters, J.; Lipsitch, M. Antibiotic resistance-the interplay between antibiotic use in animals and human beings. Lancet Infect. Dis. 2003, 3, 47-51. [CrossRef]

2. Huyghebaert, G.; Ducatelle, R.; Van Immerseel, F. An update on alternatives to antimicrobial growth promoters for broilers. Vet. J. 2011, 187, 182-188. [CrossRef] [PubMed]

3. Moghadam, M.M.; Amiri, M.; Riabi, H.R.A.; Riabi, H.R.A. Evaluation of antibiotic residues in pasteurized and raw milk distributed in the south of Khorasan-e Razavi Province, Iran. J. Clin. Diagn. Res. 2016, 10, FC31. [CrossRef] [PubMed] 
4. Martínez, J.L. Antibiotics and antibiotic resistance genes in natural environments. Science 2008, 321, 365-367. [CrossRef] [PubMed]

5. Joy, S.R.; Li, X.; Snow, D.D.; Gilley, J.E.; Woodbury, B.; Bartelt-Hunt, S.L. Fate of antimicrobials and antimicrobial resistance genes in simulated swine manure storage. Sci. Total Environ. 2014, 481, 69-74. [CrossRef] [PubMed]

6. Chen, X.; Zhang, W.; Yin, J.; Zhang, N.; Geng, S.; Zhou, X.; Jiao, X. Escherichia coli isolates from sick chickens in China: Changes in antimicrobial resistance between 1993 and 2013. Vet. J. 2014, 202, 112-115. [CrossRef] [PubMed]

7. Marshall, B.M.; Levy, S.B. Food animals and anti-microbials: Impacts on human health. Clin. Microbiol. Rev. 2011, 24, 718-733. [CrossRef] [PubMed]

8. Ministry of Agriculture and Rural Affairs of the People's Republic of China. Announcement No. 194. Available online: http://www.moa.gov.cn/gk/tzgg_1/gg/201907/t20190710_6320678.htm (accessed on 10 July 2019).

9. Filippidis, A.; Godelitsas, A.; Charistos, D.; Misaelides, P.; Kassoli-Fournaraki, A. The chemical behavior of natural zeolites in aqueous environments: Interactions between low-silica zeolites and $1 \mathrm{M} \mathrm{NaCl}$ solutions of different initial pH-values. Appl. Clay Sci. 1996, 11, 199-209. [CrossRef]

10. Mumpton, F.A.; Fishman, P.H. The application of natural zeolites in animal science and aquaculture. J. Anim. Sci. 1977, 45, 1188-1203. [CrossRef]

11. Auerbach, S.M.; Ford, M.H.; Monson, P.A. New insights into zeolite formation from molecular modeling. Curr. Opin. Colloid Interface Sci. 2005, 10, 220-225. [CrossRef]

12. Yang, L.; Liu, X.Y.; He, H.W.; Zhou, H.S.; Xie, D.M.; Wang, X. Synthesis and crystallization process of nano-sized nay zeolite. Mater. Sci. Eng. Powder Metall. 2013, 18, 270-275.

13. Arnnok, P.; Patdhanagul, N.; Burakham, R. An on-line admicellar SPE-HPLC system using CTAB-modified zeolite NaY as sorbent for determination of carbamate pesticides in water. Chromatographia 2015, 78, 1327-1337. [CrossRef]

14. Kónya, Z.; Hannus, I.; Kiricsi, I. Zeolites in the environmental protection-Decomposition of chlorofluorocarbons over zeolite catalysts. Stud. Surf. Sci. Catal. 1997, 105, 1509-1516.

15. Minato, H.; Yoshida, M.; Shibue, Y. New use of natural zeolites and clay for environmental protection and remediation of toxic metals contamination sites. Am. J. Pathol. 1999, 154, 281-289.

16. Wu, Q.J.; Wang, L.C.; Zhou, Y.M.; Zhang, J.F.; Wang, T. Effects of clinoptilolite and modified clinoptilolite on the growth performance, intestinal microflora, and gut parameters of broilers. Poult. Sci. 2013, 92, 684-692. [CrossRef]

17. Wu, Q.J.; Zhou, Y.M.; Wu, Y.N.; Wang, T. Intestinal development and function of broiler chickens on diets supplemented with clinoptilolite. Asian-Australas. J. Anim. Sci. 2013, 26, 987-994. [CrossRef]

18. Fethiere, R.; Miles, R.D.; Harms, R.H. The utilization of sodium in sodium zeolite A by broilers. Poult. Sci. 1994, 73, 118-121. [CrossRef]

19. Hu, C.H.; Xiao, K.; Jiao, L.F.; Song, J. Effects of zinc oxide supported on zeolite on growth performance, intestinal barrier function and digestive enzyme activities of Nile tilapia. Aquac. Nutr. 2014, 20, 486-493. [CrossRef]

20. Papaioannou, D.S.; Kyriakis, C.S.; Alexopoulos, C.; Tzika, E.D.; Polizopoulou, Z.S.; Kyriakis, S.C. A field study on the effect of the dietary use of a clinoptilolite-rich tuff, alone or in combination with certain antimicrobials, on the health status and performance of weaned, growing and finishing pigs. Res. Vet. Sci. 2004, 76, 19-29. [CrossRef]

21. Mercurio, M.; Cappelletti, P.; De Gennaro, B.; De Gennaro, M.; Bovera, F.; Iannaccone, F.; Grifa, C.; Langella, A.; Moretti, V.; Esposito, L. The effect of digestive activity of pig gastro-intestinal tract on zeolite-rich rocks: An in vitro study. Micropor. Mesopor. Mater. 2016, 225, 133-136. [CrossRef]

22. Kan, C.A. Factors affecting absorption of harmful substances from the digestive tract of poultry and their level in poultry products. Worlds Poult. Sci. J. 1994, 50, 39-53. [CrossRef]

23. Incharoen, T.; Khambualai, O.; Yamauchi, K. Performance and histological changes of the intestinal villi in chickens fed dietary natural zeolite including plant extract. Asian J. Poult. Sci. 2009, 3, 42-50.

24. Khambualai, O.; Ruttanavut, J.; Kitabatake, M.; Goto, H.; Erikawa, T.; Yamauchi, K. Effects of dietary natural zeolite including plant extract on growth performance and intestinal histology in Aigamo ducks. Br. Poult Sci. 2009, 50, 123-130. [CrossRef] [PubMed] 
25. Papatsiros, V.G.; Katsoulos, P.D.; Koutoulis, K.C.; Karatzia, M.; Dedousi, A.; Christodoulopoulos, G. Alternatives to antibiotics for farm animals. CAB Rev. 2013, 8, 1-15. [CrossRef]

26. Caflisch, K.M.; Schmidt-Malan, S.M.; Mandrekar, J.N.; Karau, M.J.; Nicklas, J.P.; Williams, L.B.; Patel, R. Antibacterial activity of reduced iron clay against pathogenic bacteria associated with wound infections. Int. J. Antimicrob. Agents. 2018, 52, 692-696. [CrossRef]

27. Zhao, W.; Liu, X.; Huang, Q.; Walker, S.L.; Cai, P. Interactions of pathogens Escherichia coli and Streptococcus suis with clay minerals. Appl. Clay Sci. 2012, 69, 37-42. [CrossRef]

28. Al-Nasser, A.Y.; Al-Zenki, S.F.; Al-Saffar, A.E.; Abdullah, F.K.; Al-Bahouh, M.E.; Mashaly, M. Zeolite as a feed additive to reduce Salmonella and improve production performance in broilers. Int. J. Poult. Sci. 2011, 10, 448-454. [CrossRef]

29. Zou, Y.L.; Huang, H.; Chu, M.; Sun, Y.L.; Yu, H.F.; Liu, Z.; Lin, J.W.; Yin, D.Q. Adsorption of tetracycline on natural and $\mathrm{CaCl}_{2}$-modified zeolites. J. Environ. Eng. 2012, 6, 2612-2618.

30. De Sousa, D.N.R.; Insa, S.; Mozeto, A.A.; Petrovic, M.; Chaves, T.F.; Fadini, P.S. Equilibrium and kinetic studies of the adsorption of antibiotics from aqueous solutions onto powdered zeolites. Chemosphere 2018, 205, 137-146. [CrossRef]

31. Zhang, J.; Chen, M.; Sui, Q.; Tong, J.; Jiang, C.; Lu, X.; Zhang, Y.; Wei, Y. Impacts of addition of natural zeolite or a nitrification inhibitor on antibiotic resistance genes during sludge composting. Water Res. 2016, 91, 339-349. [CrossRef]

32. Prasai, T.P.; Walsh, K.B.; Bhattarai, S.P.; Midmore, D.J.; Van, T.T.; Moore, R.J.; Stanley, D. Zeolite food supplementation reduces abundance of enterobacteria. Microbiol. Res. 2017, 195, 24-30. [CrossRef] [PubMed]

33. Mallek, Z.; Fendri, I.; Khannous, L.; Hassena, A.B.; Traore, A.I.; Ayadi, M.A.; Gdoura, R. Effect of zeolite (clinoptilolite) as feed additive in Tunisian broilers on the total flora, meat texture and the production of omega 3 polyunsaturated fatty acid. Lipids Health Dis. 2012, 11, 35. [CrossRef] [PubMed]

34. Suchý, P.; Strakova, E.; Večerek, V.; Klouda, Z.; Kráčmarová, E. The effect of a clinoptilolite-based feed supplement on the performance of broiler chickens. Czech J. Anim. Sci. 2006, 51, 168-173. [CrossRef]

35. Elliot, M.A.; Edwards, H.M., Jr. Comparison of the effects of synthetic and natural zeolite on laying hen and broiler chicken performance. Poult. Sci. 1991, 70, 2115-2130. [CrossRef] [PubMed]

36. Cheng, Y.F.; Chen, Y.P.; Chen, R.; Su, Y.; Zhang, R.Q.; He, Q.F.; Zhou, Y.M. Dietary mannan oligosaccharide ameliorates cyclic heat stress-induced damages on intestinal oxidative status and barrier integrity of broilers. Poult. Sci. 2019. [CrossRef]

37. Li, J.; Cheng, Y.; Chen, Y.; Qu, H.; Zhao, Y.; Wen, C.; Zhou, Y. Dietary chitooligosaccharide inclusion as an alternative to antibiotics improves intestinal morphology, barrier function, antioxidant capacity, and immunity of broilers at early age. Animals 2019, 9, 493. [CrossRef] [PubMed]

38. Livak, K.J.; Schmittgen, T.D. Analysis of relative gene expression data using real-time quantitative PCR and the $2^{-\Delta \Delta C T}$ method. Methods 2001, 25, 402-408. [CrossRef]

39. Zhu, Y.G.; Johnson, T.A.; Su, J.Q.; Qiao, M.; Guo, G.X.; Stedtfeld, R.D.; Hashsham, S.A.; Tiedje, J.M. Diverse and abundant antibiotic resistance genes in Chinese swine farms. Proc. Natl. Acad. Sci. USA 2013, 110, 3435-3440. [CrossRef]

40. Shurson, G.C.; Ku, P.K.; Miller, E.R.; Yokoyama, M.T. Effects of zeolite or clinoptilolite in diets of growing swine. J. Anim. Sci. 1984, 59, 1536-1545. [CrossRef]

41. Pond, W.G.; Mumpton, F.A. Zeo-Agriculture: Use of Natural Zeolites in Agriculture and Aquaculture; Westview Press: Boulder, CO, USA, 1984.

42. Parisini, P.; Martelli, G.; Sardi, L.; Escribano, F. Protein and energy retention in pigs fed diets containing sepiolite. Anim. Feed Sci. Technol. 1999, 79, 155-162. [CrossRef]

43. Miazzo, R.; Rosa, C.A.R.; De Queiroz Carvalho, E.C.; Magnoli, C.; Chiacchiera, S.M.; Palacio, G.; Dalcero, A. Efficacy of synthetic zeolite to reduce the toxicity of aflatoxin in broiler chicks. Poult. Sci. 2000, 79, 1-6. [CrossRef] [PubMed]

44. Girish, C.K.; Smith, T.K. Effects of feeding blends of grains naturally contaminated with Fusarium mycotoxins on small intestinal morphology of turkeys. Poult. Sci. 2008, 87, 1075-1082. [CrossRef] [PubMed]

45. Liu, M.; Gao, R.; Meng, Q.; Zhang, Y.; Bi, C.; Shan, A. Toxic effects of maternal zearalenone exposure on intestinal oxidative stress, barrier function, immunological and morphological changes in rats. PLoS ONE 2014, 9, e106412. [CrossRef] [PubMed] 
46. Przybylska-Gornowicz, B.; Tarasiuk, M.; Lewczuk, B.; Prusik, M.; Ziółkowska, N.; Zielonka, Ł.; Gajęcki, M.; Gajęcka, M. The effects of low doses of two Fusarium toxins, zearalenone and deoxynivalenol, on the pig jejunum. A light and electron microscopic study. Toxins 2015, 7, 4684-4705. [CrossRef]

47. Wawrzyniak, A.; Kapica, M.; Stępień-Pyśniak, D.; Szewerniak, R.; Olejarska, A.; Jarosz, Ł. Effect of feeding transcarpathian zeolite on gastrointestinal morphology and function in broiler chickens. Br. J. Poult. Sci. 2017, 19, 737-746. [CrossRef]

48. Chen, Y.P.; Cheng, Y.F.; Li, X.H.; Zhang, H.; Yang, W.L.; Wen, C.; Zhou, Y.M. Dietary palygorskite supplementation improves immunity, oxidative status, intestinal integrity, and barrier function of broilers at early age. Anim. Feed Sci. Technol. 2016, 219, 200-209. [CrossRef]

49. Su, Y.; Chen, Y.P.; Cheng, Y.F.; Wen, C.; Zhou, Y.M. Effects of modified palygorskite supplementation on egg quality and mineral element content, and intestinal integrity and barrier function of laying hens. Biol. Trace Elem. Res. 2018, 186, 529-537. [CrossRef]

50. Tatar, A.; Boldaji, F.; Dastar, B.; Yaghobfar, A. Comparison of different levels of zeolite on serum characteristics, gut $\mathrm{pH}$, apparent digestibility of crude protein and performance of broiler chickens. In Proceedings of the International Zeolite Conference, Tehran, Iran, 29 April-1 May 2008; Volume 235.

51. Ly, J.; Grageola, F.; Lemus, C.; Castrp, M. Ileal and rectal digestibility of nutrients in diet based on leucaena for pigs, influence of the inclusion zeolite. J. Anim. Vet. Adv. 2007, 6, 1371-1376.

52. Yu, B.P. Cellular defenses against damage from reactive oxygen species. Physiol. Rev. 1994, 74, $139-162$. [CrossRef]

53. Birben, E.; Sahiner, U.M.; Sackesen, C.; Erzurum, S.; Kalayci, O. Oxidative stress and antioxidant defense. World Allergy Organ. J. 2012, 5, 9-19. [CrossRef]

54. Ayala, A.; Muñoz, M.F.; Argüelles, S. Lipid peroxidation: Production, metabolism, and signaling mechanisms of malondialdehyde and 4-hydroxy-2-nonenal. Oxid. Med. Cell Longev. 2014, 2014, 360-438. [CrossRef] [PubMed]

55. Çakmaz, R.; Büyükaşık, O.; Kahramansoy, N.; Erkol, H.; Çöl, C.; Boran, Ç.; Buğdaycı, G. A combination of plasma DAO and citrulline levels as a potential marker for acute mesenteric ischemia. Libyan J. Med. 2013, 8, 20596. [CrossRef] [PubMed]

56. Alexander, C.; Rietschel, E.T. Invited review: Bacterial lipopolysaccharides and innate immunity. J. Endotoxin Res. 2001, 7, 167-202. [CrossRef] [PubMed]

57. Sun, X.Q.; Fu, X.B.; Rong-Zhang, Y.L.; Deng, Q.; Jiang, X.G.; Sheng, Z.Y. Relationship between plasma D (-)-lactate and intestinal damage after severe injuries in rats. World J. Gastroenterol. 2001, 7, 555-558. [CrossRef]

58. Luk, G.D.; Bayless, T.M.; Baylin, S.B. Diamine oxidase (histaminase): A circulating marker for rat intestinal mucosal maturation and integrity. J. Clin. Investig. 1980, 66, 66-70. [CrossRef]

59. Li, J.Y.; Lu, Y.; Hu, S.; Sun, D.; Yao, Y.M. Preventive effect of glutamine on intestinal barrier dysfunction induced by severe trauma. World J. Gastroenterol. 2002, 8, 168-171. [CrossRef]

60. Lerner, A.; Matthias, T. Changes in intestinal tight junction permeability associated with industrial food additive sex plain the rising incidence of autoimmune disease. Autoimmun. Immun. Rev. 2015, 14, 479-489. [CrossRef]

61. Chalvatzi, S.; Kalamaki, M.S.; Arsenos, G.; Fortomaris, P. Dietary supplementation with the clay mineral palygorskite affects performance and beneficially modulates cecal microbiota in laying pullets. J. Appl. Microbiol. 2016, 120, 1033-1040. [CrossRef]

62. Zhou, P.; Tan, Y.Q.; Zhang, L.; Zhou, Y.M.; Gao, F.; Zhou, G.H. Effects of dietary supplementation with the combination of zeolite and attapulgite on growth performance, nutrient digestibility, secretion of digestive enzymes and intestinal health in broiler chickens. Asian-Australas. J. Anim. Sci. 2014, 27, 1311. [CrossRef]

63. Slamova, R.; Trckova, M.; Vondruskova, H.; Zraly, Z.; Pavlik, I. Clay minerals in animal nutrition. Appl. Clay Sci. 2011, 51, 395-398. [CrossRef]

64. Zhang, J.; Lv, Y.; Tang, C.; Wang, X. Effects of dietary supplementation with palygorskite on intestinal integrity in weaned piglets. Appl. Clay Sci. 2013, 86, 185-189. [CrossRef]

(C) 2019 by the authors. Licensee MDPI, Basel, Switzerland. This article is an open access article distributed under the terms and conditions of the Creative Commons Attribution (CC BY) license (http://creativecommons.org/licenses/by/4.0/). 